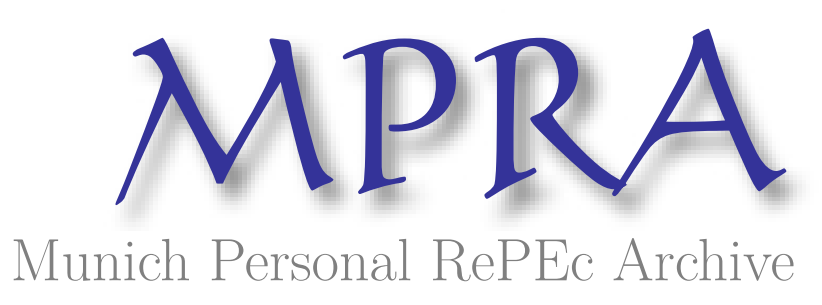

Impact of playoffs on seasonal uncertainty in Czech ice hockey Extraliga

Lahvicka, Jiri

27 February 2013

Online at https://mpra.ub.uni-muenchen.de/44608/

MPRA Paper No. 44608, posted 01 Mar 2013 11:50 UTC 


\title{
IMPACT OF PLAYOFFS ON SEASONAL UNCERTAINTY IN CZECH ICE HOCKEY EXTRALIGA
}

\author{
JIŘí LAHVIČKA
}

\section{E-MAIL: JIRKA@LAHVICKA.CZ}

In the top Czech ice hockey competition "Extraliga", 14 geographically close teams compete during a regular season in a pure round-robin tournament. However, the eventual champion is determined in the additional playoff stage; the regular season just decides which teams qualify for the playoffs and how these teams are seeded. This paper uses a Monte Carlo simulation to show that although the additional playoff stage heavily favors higher-seeded teams and consists of a lot of matches, it lowers the probability of the best team becoming a champion and thus increases seasonal uncertainty.

Keywords: seasonal uncertainty, Monte Carlo, ice hockey, tournament design

JEL classification: C53; L83

Last revision: February 24th 2013 


\section{INTRODUCTION}

One of the most important results of sports economics is the observation that a tighter competition with a more uncertain outcome will attract more spectators. This so-called uncertainty of outcome hypothesis was first formulated by Rottenberg (1956), who noted that a baseball team winning too many games would attract fewer spectators, and later expanded on by Neale (1964), who claimed that a sports league will attract higher attendances if league standings are close and change often.

The sports economics literature distinguishes three different types of uncertainty of outcome (Szymanski 2003, García and Rodríguez 2009) - match uncertainty (how certain the result of one specific match is), seasonal uncertainty (how certain the competition winner and other similar outcomes are), and championship uncertainty (whether there is a long-run domination by one team). Instead of seasonal or championship uncertainty, some authors use the term competitive balance; however, Scarf et al. (2008) make a useful distinction between competitive balance, which is defined as relative strengths of competing teams, and uncertainty of outcome, which also depends on tournament design. Of course, all these concepts are related; higher competitive balance (more evenly matched teams) leads to higher match uncertainty, seasonal uncertainty, and championship uncertainty. Similarly, a shorter competition with fewer matches would typically lead to higher seasonal uncertainty; this would redistribute prize money and other types of revenues to weaker teams, reduce teams' incentives to invest into stronger players, and thus potentially increase competitive balance.

The relationship between match uncertainty and attendance has a mixed empirical support; for example, Buraimo and Simmons (2008) found that increased match uncertainty actually lowered match attendance in English Premier League; a similar result was obtained by Coates and Humphreys (2011) for NHL. On the other hand, empirical evidence mostly confirms the uncertainty of outcome hypothesis for seasonal and championship uncertainty - see Szymanski (2001) for English Premier League, Humphreys (2002) for American MLB, Pawlowski and Budzinski (2012) for three major European soccer leagues, or Szymanski (2003) for an overview of multiple studies. The uncertainty of outcome hypothesis also seems to be accepted by many competition organizers, who implement various mechanisms purported to redistribute resources and increase competitive balance, such as TV and gate revenue sharing, payroll caps, or giving weaker teams earlier draft picks. However, sports economists have extensively analyzed these mechanisms and are generally quite skeptical about their efficiency and true goals (Vrooman 1995, Szymanski 2001, Szymanski 2003, Szymanski and Késenne 2004).

Another obvious way available to competition organizers to increase the uncertainty of outcome is modifying the tournament design. There are two basic tournament types used in team sports competitions - a round-robin tournament, where each team plays the same number of matches against every other team (e.g. English Premier League), and a knock-out tournament, where teams are paired together, winners progress to the next round and losers are eliminated, until there is only 
one team left (e.g. English FA Cup or NHL playoffs). By modifying these tournament types (such as changing the total number of matches between each team pair) or by combining them together in various ways (e.g. FIFA World Cup finals, where a group stage is followed by the playoffs), the competition organizers can directly influence the probability that the best team wins, i.e. seasonal uncertainty. Of course, the tournament design choice is constrained by many factors such as the number of teams (round-robins are impractical for a large number of teams) or the time available for the competition.

The relationship between seasonal uncertainty and a specific tournament design choice has come into focus only recently. Scarf et al. (2008) compared various designs for the UEFA Champions League (32 team home and away round-robin, 32 team pure 2 leg knock-out with or without seeding, various combinations of group rounds and knock-out) using a Monte Carlo simulation approach. The round-robin design (which would be extremely impractical in reality due to a large number of matches) maximized the probability of the best team winning the tournament, while the unseeded 2 leg design maximized the uncertainty of outcome. A similar approach was used in Scarf and Yusof (2011) to show that seeding favors stronger teams and thus reduces uncertainty of outcome in FIFA World Cup finals.

This paper uses an approach similar to Scarf et al. (2008) to analyze the tournament design of the top Czech ice-hockey competition "Extraliga". The Extraliga design is rather peculiar; there are currently just 14 geographically close teams that compete during the regular season in a pure round-robin tournament. However, the eventual competition champion is determined in the additional NHL-like playoff stage and the regular season round-robin tournament is just used to decide which teams qualify for the playoffs and how these teams are seeded. It is important to note that the additional playoff stage is not really necessary to determine the competition winner; in fact, before the 1985/86 season, the round-robin tournament winner was declared the champion and the season ended without any playoffs. Another interesting observation is that in the last six seasons (2006/07-2011/12), there were six different regular season winners and five different playoffs winners with the same team winning both in only one season, so the seasonal and championship uncertainties in the Extraliga seem to be very high. This raises two related questions: First, does the best team have a higher probability of winning the regular season or the additional playoff stage? Second, how does adding the playoff stage impact the probabilities of all the other teams that they become a new champion?

To analyze these questions, this paper uses a Monte Carlo simulation based on six different sets of realistic team strengths derived from the actual results of six Extraliga seasons (2006/07-2011/12). The simulation results show that although the additional playoff stage heavily favors teams that placed better in the regular season and consists of quite a lot of matches, it lowers the probability that the best team becomes the champion (especially if this team is very dominant) and raises this probability for weaker teams (especially if they are significantly weaker than the best team, but still above average). This is also true for some obvious modifications of the playoffs. Therefore, the addition of the playoffs to the regular Extraliga season increases seasonal uncertainty. 
The rest of the paper is organized as follows: Section 2 overviews the Extraliga tournament design and compares it to other competitions; Section 3 describes the individual match model, how team strengths are estimated from actual results, and how the whole season is simulated; Section 4 presents the simulation results; and Section 5 concludes. 


\section{EXTRALIGA OVERVIEW}

The Czech ice hockey Extraliga was established in season 2003/04 (after Czechoslovakia split into the Czech Republic and the Slovak Republic) and is currently the most popular team sports competition in the Czech Republic. ${ }^{1}$ Although the specific rules changed several times, the basic tournament design has stayed the same. First, all teams compete in a round-robin tournament that decides which teams qualify for the playoff stage and how they are seeded; second, the playoffs are used to determine the champion and all other final rankings. The same two-part tournament design was also regularly used in former Czechoslovakia since the 1985/86 season and experimented with in the 1970s. This section describes the competition rules that were first implemented in season 2006/07, were in place during all six seasons analyzed in this paper (2006/07-2011/12), and are still valid as of February 2013.

The Extraliga consists of 14 teams. In the regular season, which typically runs from September to March, each team plays two home and two away matches against all the other teams $(4 \times 13=52$ matches in total). Each ice hockey match consists of three 20-minute thirds (so-called regulation time). The team scoring more goals is the winner and receives 3 points, while the losing team gets 0 points. A draw is not possible - if a match is undecided in the regulation time, it goes into extra time, which lasts either 5 minutes or until a goal is scored. If the match is not decided in the extra time, a penalty shootout determines which team is considered to have scored the decisive goal. The extra time/penalty shootout winner receives 2 points, while the losing team gets 1 point. In the final regular season league table, teams are ranked according to the following criteria (in that order): total points, points from head-on matches against teams with the same number of total points, score difference in these head-on matches, total score difference, and total number of goals scored. Since the 2009/10 season, the regular season winner actually receives a minor trophy (the President's Cup); before, there was no trophy at all. After the regular season, all teams play at least several additional matches - the top 10 teams qualify for the playoffs, while the bottom 4 teams proceed to the play-out stage (not relevant for this paper) that determines which team has to defend its Extraliga spot against a lower competition winner.

The playoff stage, which usually takes place in March and April, consists of four rounds - the preliminary round, the quarterfinals, the semifinals, and the final. In the preliminary round, teams that finished $7^{\text {th }}-10^{\text {th }}$ in the regular season compete for two spots in the quarterfinals, where they are joined by the top 6 teams. In each round, teams are seeded according to their regular season final rank and paired so that the highest surviving seed plays against the lowest surviving seed, the

\footnotetext{
${ }^{1}$ In the 2011/12 season, the total Extraliga attendance was about 2.2 million spectators, while the regular season alone attracted almost 1.8 million spectators. In the same season, the top soccer competition "Gambrinus liga" attracted only a bit over 1.1 million spectators. The average regular season match attendance was 4,824 for the Extraliga and 4,710 for the Gambrinus liga. Sources: hokej.cz, fotbal.idnes.cz (both accessed on February 23rd, 2013).

2 The rules were compiled from the following websites: cslh.cz (Czech Ice Hockey Association), hokej.cz, and avlh.sweb.cz (Archive of Ice Hockey Results); all websites were accessed on February 15 ${ }^{\text {th }}, 2013$.
} 
second-highest seed plays against the second-lowest seed and so on. Each pair of teams plays a bestof-five (preliminary round) or best-of-seven (all the other rounds) series of matches, so the first team to defeat their opponent three (preliminary round) or four times (all the other rounds) proceeds to the next round. In each series of matches, the higher-ranked team plays the first, second, fifth, and seventh match on its home ice. If a match is tied, the extra time lasts 10 minutes instead of 5. In the fifth (preliminary round), or seventh (all the other rounds), penalty shootouts are not possible and any extra time lasts until a goal is scored.

The Extraliga playoff stage is quite similar to the system used in the top two ice hockey club competitions in the world - the NHL (USA) and KHL (Russia and other countries). However, the regular season in these two competitions is different; the participating teams are split into groups according to their geographical location and play more matches against geographically close teams. Since teams are not grouped according to their strengths, groups are not designed to be balanced and the regular season winner is not clear (though it can be determined based on the overall record). Therefore, the playoff stage used in the NHL and KHL is somewhat of a necessity due to large distances between teams, but seems superfluous in a small country such as the Czech Republic. It is also interesting that the additional playoff stage is practically nonexistent in European soccer competitions, but used in the MLS (top USA soccer competition). On the other hand, other top European ice hockey competitions (e.g. in Sweden, Finland, Germany or the Slovak Republic) are organized very similarly to the Czech Extraliga and do use playoffs. It can be concluded that for small countries, adding a playoff stage to a pure round-robin tournament is simply a design choice, not a necessity. The question is - what is the impact of this design choice on seasonal uncertainty in general and on chances of the strongest team in particular?

At first sight, the Extraliga playoff stage should be quite good at identifying the best team. First, each pair of teams plays up to seven matches to determine which team moves to the next round; this is much more than one or two matches typically used in soccer. Second, teams are reseeded for each round; this type of seeding was shown to help the strongest teams the most by Scarf and Yusof (2011). Third, higher-seeded teams play any decisive match in the series on their home ice. However, the regular-season round-robin tournament consists of a high number of matches as well, so it also seems to be suitable for determining which team is the best. Clearly, a more detailed analysis is needed to decide which type of tournament design favors which teams. 


\section{MODEL}

The impact of the playoffs on seasonal uncertainty could be investigated by simply looking at what has happened historically in both the regular season and the playoff stage. On example of such a study is Szemberg et al. (2012), who analyzed eight top ice hockey competitions and showed that the regular season winner won the playoff stage in just $43 \%$ of the cases. However, this approach has three drawbacks - first, the dataset is either too small or must include different leagues with different rules in different time periods; second, it is not possible to find out how exactly the additional playoff stage impacts the championship chances of different teams; third, it is not possible to analyze various what-if scenarios, such as the relationship between the final regular season rank and the probability of winning the playoffs keeping the team strength constant.

Therefore, this paper uses a three-step method similar to Scarf et al. (2008). First, actual results of six different regular Extraliga seasons are used to estimate six sets of team strengths. Second, these six sets of team strengths are used to simulate 1,000,000 times each of the six corresponding seasons including the playoff stage down to the level of an individual match score; the actual season results are then used to verify that these simulations are realistic. Third, the huge resulting dataset consisting of 6,000,000 completed simulations is used to investigate the impact of the playoffs on seasonal uncertainty.

To generate an individual match score between any two teams, this paper employs one of several methods introduced by Maher (1982), but modified for ice hockey. The unmodified method assumes that each team's strength can be described by four parameters - attack strength in home matches (HomeAttack), attack strength in away matches (AwayAttack), defense strength in home matches (HomeDefense), and defense strength in away matches (AwayDefense). For attack strengths, a higher number is better, while for defense strengths, a lower number is better. If a team $i$ plays at home against team $j$, the score is composed of two random numbers drawn from two independent Poisson distributions with expected values of HomeAttack ${ }_{i}^{*}$ AwayDefense $_{j}$ (goals scored by the home team)

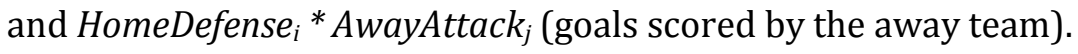

To be able to simulate any possible match in a season, it is necessary to somehow set 56 parameter values (14 teams x 4 strength parameters per team). It would be possible to randomly generate one or more sets of these parameters, but they would not necessarily correspond to a typical team strength distribution in a season. A better solution is to estimate the parameters based on actual results (Maher 1982, Scarf and Yusof 2011). In this paper, the actual results of six Extraliga seasons (2006/07-2011/12) are used to estimate six realistic sets of parameters. For each season, this is done by setting the total expected numbers of regulation-time goals scored and conceded by each 
team in its home and away matches equal to the corresponding actual values in a given season ${ }^{3}$ and solving the resulting system of equations (for details, see Appendix A: Estimating team strengths).

The simple model described above does not take into account two factors specific to ice hockey first, a team trailing by one goal towards the end of the match usually plays much more aggressively and eventually replaces their goaltender with another attacking player (so-called power play), thus dramatically increasing chances of both teams to score; second, a tied match does not end, but goes into extra time (possibly followed by a penalty shootout).

To model the power play option, the model is modified in the following way. First, the home team $i$ scores a random number of goals drawn from a Poisson distribution with an expected value of $7 / 8^{*}$ HomeAttack $_{i}{ }^{*}$ AwayDefense $_{j}$ and the away team scores a random number of goals drawn from a Poisson distribution with an expected value of $7 / 8 *$ HomeDefense $_{i}{ }^{*}$ AwayAttack $_{j}$ (this represents the score several minutes before the end of the match). If neither team is trailing by one goal, the regulation time score stays unchanged. If the home team $i$ trails by one goal, it scores an additional Poisson-distributed number of goals with an expected value of $3 / 10{ }^{*}$ HomeAttack $_{i}{ }^{*}$ AwayDefense $_{j}$ and the away team $j$ scores an additional Poisson-distributed number of goals with an expected value of $5 / 10 *$ HomeDefense $_{i}{ }^{*}$ AwayAttack $_{j}$. Similarly, if the home team $i$ leads by one goal, it scores an additional Poisson distributed number of goals with an expected value of 5/10 * HomeAttack $_{i}^{*}$ AwayDefense $_{j}$ and the away team $j$ scores an additional Poisson-distributed number of goals with an expected value of $3 / 10 *$ HomeDefense $_{i} *$ AwayAttack $_{j}$. These expected values for last-minute goals are quite high and strongly favor the leading team, but they reflect two observations about ice hockey matches made by Thomas (2007); first, the average number of goals per minute sharply increases in the last two minutes; second, if a goal is scored during power play, it is about twice as likely to be scored by a leading team. This power play modification is also calibrated so that it does not change the expected number of goals scored by each team compared to the unmodified model this means that the estimated strength parameters are still valid.

To model the extra time, it is simply assumed that if a match is tied after regulation time, an extra time/penalty shootout winning goal will be scored by the home team $i$ with the probability of HomeAttack $_{i}$ *AwayDefense / $_{j}\left(\right.$ HomeAttack $_{i}{ }^{*}$ AwayDefense $_{j}+$ HomeDefense $_{i}$ * AwayAttack $_{j}$ ) and by the away team $j$ with the probability of HomeDefense ${ }_{i}^{*}$ AwayAttack $_{j} /\left(\right.$ HomeAttack $_{i} *$ AwayDefense $_{j}+$ HomeDefense $_{i} *$ AwayAttack $_{j}$ ).

In the next step, it is necessary to get from the estimated strength parameters of all teams in a given season and the individual match model to probabilities of a given team winning the regular season or the playoffs. Due to the high number of matches and the complicated tournament design, the only feasible option is the Monte Carlo approach. First, results of all regular season matches are randomly generated and points are assigned. Second, these results are used to put together the final table (using all applicable ranking criteria) and decide which teams qualify for the playoff stage. Third, the teams are seeded and paired and all corresponding playoff matches are played until there

${ }^{3}$ All actual season data were gathered from the websites hokej.cz, and avlh.sweb.cz (Archive of Ice Hockey Results); both websites were accessed on January $18^{\text {th }}, 2013$. 
is a competition champion. This process is repeated 1,000,000 times for each set of strength parameters. In the end, there are 6,000,000 completed simulations corresponding to six actual seasons. An ex ante probability of any scenario in any season is then approximated by the relative frequency of this scenario in corresponding simulations. Because the number of simulations is very high, the estimated probabilities are very close to the exact probabilities that could (in theory) be obtained by solving the model analytically. ${ }^{4}$

Although there are some possible improvements to predicting individual matches (Maher 1982, Dixon and Coles 1997, Rue and Salvesen 2000, Goddard 2005), the model as a whole is already quite realistic. This can be shown by comparing the aggregate simulation statistics against the corresponding actual results. Specifically, there are no significant differences between the simulated and actual total number of regular season goals (including extra time); the simulated and actual relative frequencies of home/away regulation/extra time wins; the simulated and actual minimum and maximum points in a given season; and the simulated and actual relative frequencies of playoff series results (all descriptive statistics and statistical tests are provided in Appendix B: Model verification). Of course, the model also has some limitations. Two factors not included in the model are short-term strength fluctuations (caused, for example, by injuries of key players) and a possible strategy mentioned in Szemberg et al. (2012) of a team expending less effort during the regular season to have more energy for the playoff stage. The impact of both these factors is discussed in the next section.

${ }^{4}$ For example, the probability that a given team in a given season wins the regular season or the playoffs is estimated with a standard error less than 0.0005 . 


\section{RESUlts}

To investigate the impact of the additional playoff stage on seasonal uncertainty, the dataset of completed simulations of six seasons is used to estimate two probabilities for each team in each season - the probability of winning the regular season and the probability of winning the playoffs. Since there are 14 teams per season, there are $6 * 14=84$ pairs of probabilities. Based on these probabilities, it is possible to determine the best team in each season - it is simply the team with the highest probability of winning the regular season. Similarly, the second best team is the team with the second highest probability of winning the regular season and so on. It is important to note that if the teams are ordered by the probability of winning the playoffs instead, the ordering is identical for top 4 and bottom 4 teams in every season and very similar otherwise. Simply said, succeeding in the playoffs requires the same skills as succeeding in the regular season (at least in the presented model). 5

The 84 pairs of probabilities (one pair for each team in each season) are represented by points in Figure 1 . The best team in each season is marked by a bigger and darker point. On the 45-degree line, the probability of winning the regular season equals the probability of winning the playoffs.

\footnotetext{
5 Theoretically, an average team that would become stronger in away matches and weaker in home matches could keep their regular season chances constant, while increasing their chances in the playoffs; however, this seems to have a negligible impact in the dataset.
} 


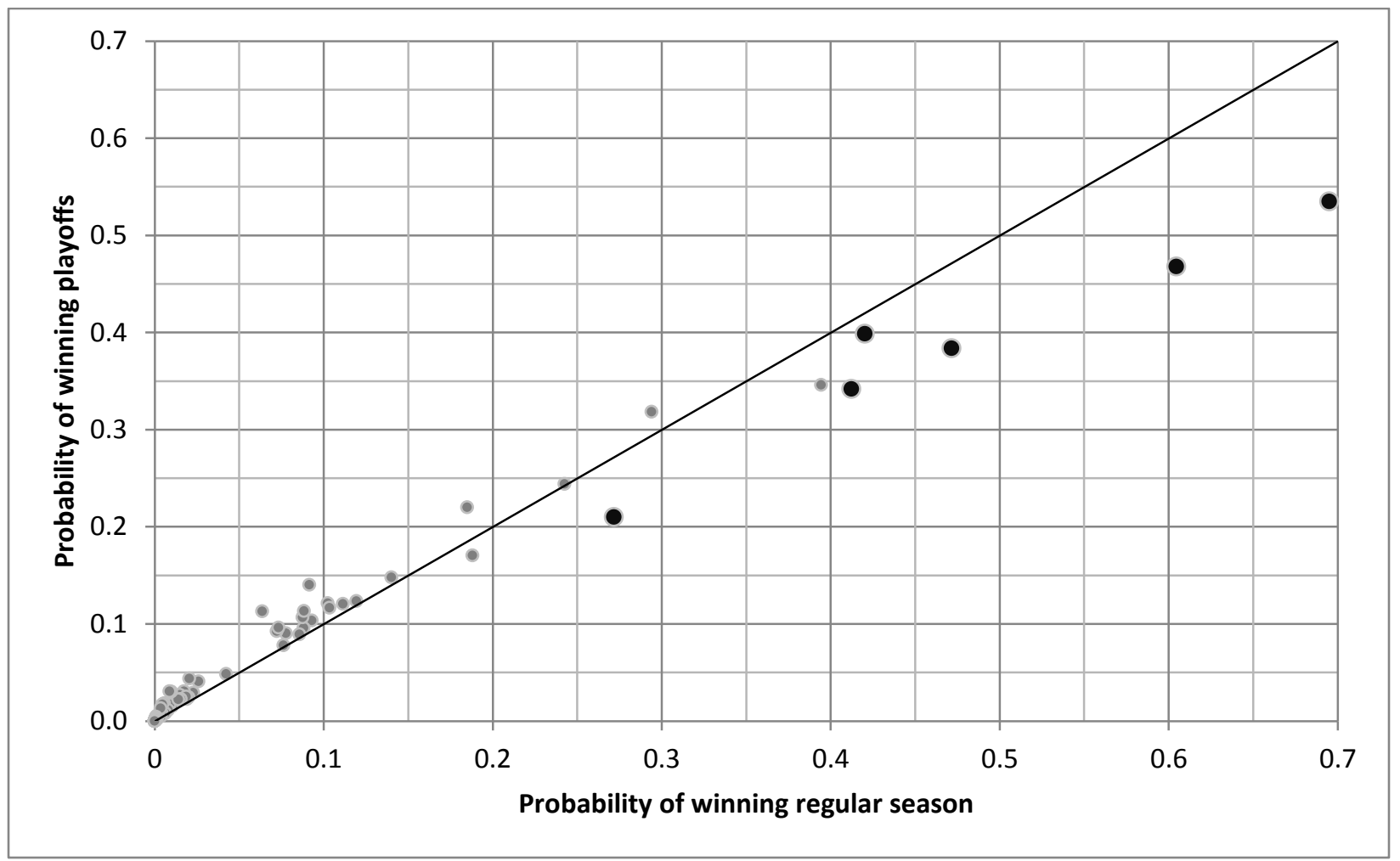

FigURE 1: PROBABILITY OF WINNING REgULAR SEASON VS. PLAYOFFS, BEST TEAMS IN EACH SEASON IN BOLD

The first obvious observation is that that all six points representing the best teams in each season are below the 45-degree line, so the additional playoff stage decreases their probability of becoming the champion. The difference is especially large for very dominant teams - Sparta Praha in the 2011/12 season (represented by the rightmost point) had a 69.5 percent chance of winning the regular season (and did actually win), but just a 53.5 percent chance of winning the playoffs (and did not actually win), so the additional playoff stage decreased their probability of becoming a champion by 16 percentage points. On the other hand, all points representing weaker teams (less than 15 percent probability of winning the regular season) are above the 45 -degree line, so such teams' chances of becoming a champion are helped by the additional playoff stage.

To analyze the change in championship probability from adding the playoff stage in more detail, it is useful to look at these changes for the best team in each season, the second-best team, the third-best team and so on. The maximum (bar top), average (black line), and minimum (bar bottom) changes for each level of team strength are presented in Figure 2. 


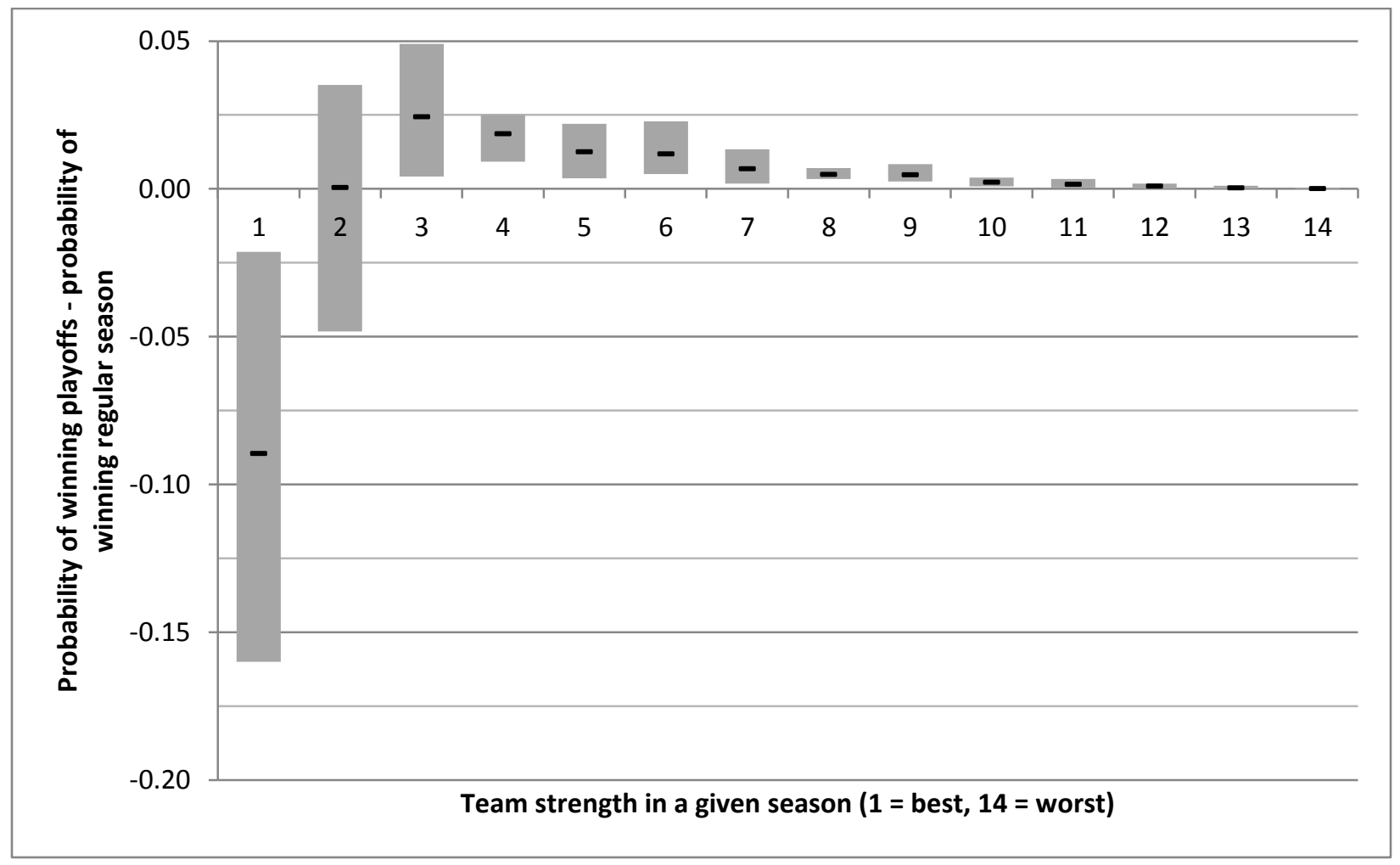

FIGURE 2: RELATIVE TEAM STRENGTH VS. CHANGE IN CHAMPIONSHIP PROBABILITY FROM ADDING PLAYOFFS

The graph shows that the additional playoff stage always decreased the championship probability of the best team in the analyzed dataset (on average by 9 percentage points), sometimes helped and sometimes hurt the second-best team, ${ }^{6}$ and always helped all the other, weaker teams. The teams that benefited the most were the third-best to sixth-best teams (much weaker than the best team, but still above average). On the other hand, the worst teams were only negligibly affected, since their probability of winning would be close to zero under any reasonable tournament design.

To summarize, the additional playoff stage decreases the championship chances of the best team (especially if it is very dominant), increases the championship chances for the other teams (especially if they are significantly weaker than the best team, but still above average), and thus increases the seasonal uncertainty. A very similar pattern emerges for different individual match models, different team strength distributions, ${ }^{7}$ or when comparing the probabilities of finishing in the top 2 in the regular season against the probabilities of reaching the playoff final. The additional

6 The second-best team was helped when being much weaker than the best team and hurt when being just a little weaker than the best team.

${ }^{7}$ A simplified individual match model without the power play option and giving each team 50\% probability of winning any extra time underestimates the number of matches decided in regulation time, but leads to almost identical championship probabilities. Increasing the strength of the best team in each season (by multiplying both attack strength parameters and dividing both defense strength parameters by a number greater than one) confirms that as a team becomes more dominant, the playoff stage decreases its championship chances by more percentage points. 
playoff stage also increases seasonal uncertainty measured by Herfindahl-Hirschman Index or Gini coefficient. ${ }^{8}$ As said in the previous section, the model does not take into account short-term strength fluctuations; however, these are likely to further increase the impact of the playoffs on seasonal uncertainty - if the best team loses several matches in a row, it does not mean much during the regular season (there are plenty of other matches to compensate), but it would likely mean an instant elimination during the playoff stage.

A natural question is why the playoffs increase seasonal uncertainty, especially considering that the combination of reseeding before each round and home ice advantage should strongly favor teams that do better in the regular season and are therefore seeded higher. A logical question is how big this advantage for stronger teams actually is.

Szemberg et al. (2012) observed that higher-seeded teams tend to win the playoffs much more often. However, this would happen even if the playoff stage design did not favor higher-seeded teams at all, since higher-seeded teams also tend to be stronger. To determine how the regular season final rank influences the probability of winning the playoffs, it is therefore necessary to keep the team strength constant. This is impossible based on just observational data, but easy using the simulation approach; for each team in each season, the probability of winning the playoffs given a particular regular season final rank can be simply estimated as the relative frequency of winning the playoffs in a subset of simulations where the team reached that rank. Figure 3 shows these probabilities of winning the playoffs conditional on a specific seed for four selected teams (dotted lines) and averaged across all teams and seasons (solid line), while bars represent simulated probabilities of a given seed winning the playoffs in the whole dataset (no matter which specific team it is).

${ }^{8}$ See Humphreys (2002) for an overview of these measures as applied to uncertainty of outcome. 


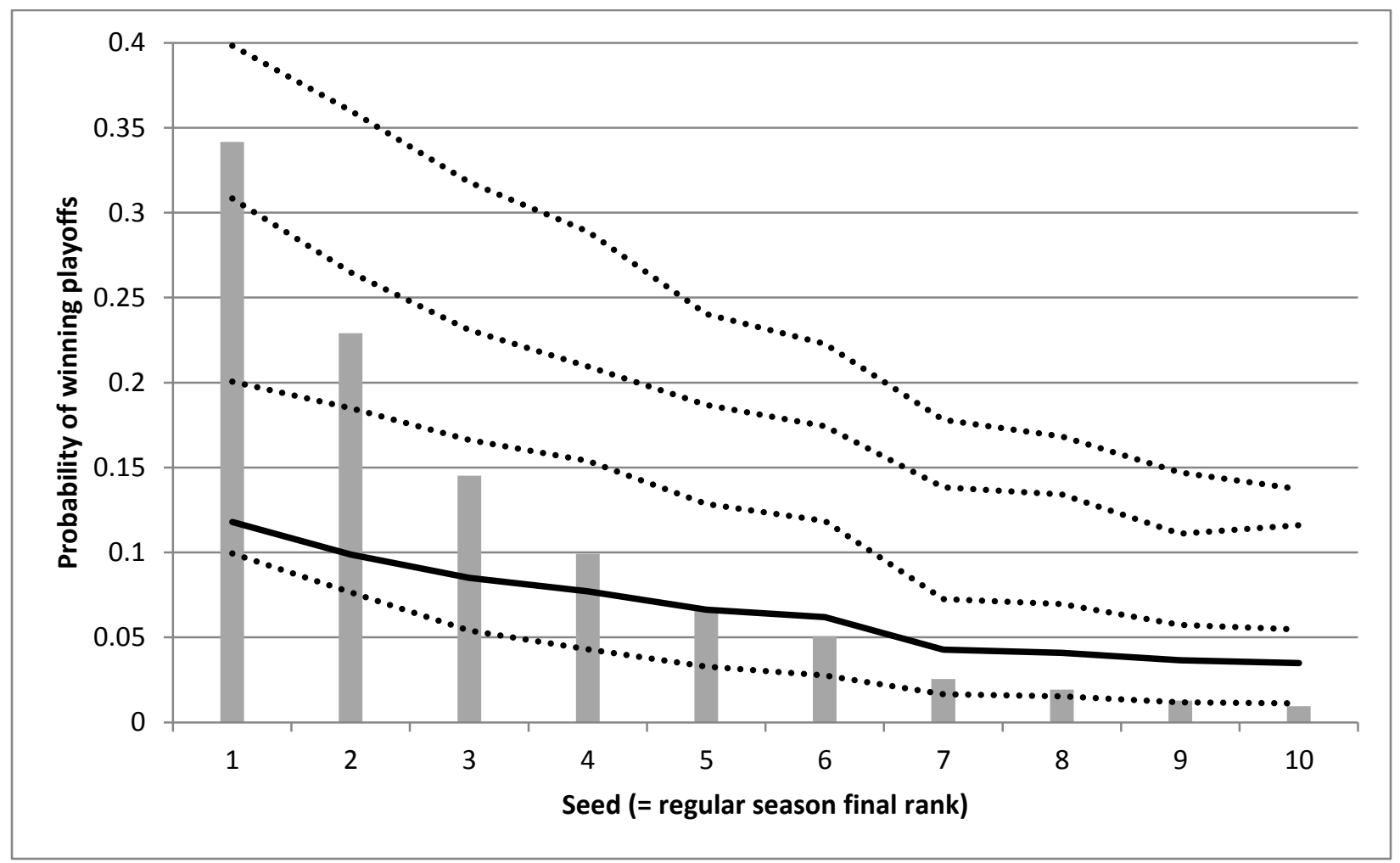

FigURE 3: PRobABILITY OF WINNING PLAYOFFS GIVEN A SPECIFIC SEED

It is obvious that as the seed gets worse, the championship probability goes gown much more slowly when controlling for team strength, so the observed pattern of higher-seeded teams winning much more often can be mostly explained by these teams simply being stronger. Nevertheless, a better regular season result still provides a significant advantage when keeping the team strength constant; on average, obtaining the best seed roughly triples the championship probability compared to the worst seed. Consequently, the potential strategy of expending less effort during the regular season to have more energy for the playoffs mentioned in the last section does not seem reasonable, especially considering that finishing in top 6 means avoiding the preliminary round and thus having about one and a half weeks of rest before the quarterfinals.

Based on the analysis above, it is clear that the playoff stage design actually does heavily favor higher-seeded teams. To understand why it still decreases the probability that the best team wins, it is helpful to go back to the example of Sparta Praha in season 2011/12. As mentioned above, Sparta Praha had a 69.5 percent probability of winning the regular season and a 53.5 percent probability of winning the playoffs. The probability of Sparta Praha winning the playoffs can be expressed as a product of four different numbers - they had a 99.97 percent probability of qualifying for the quarterfinals (either directly or from the preliminary round); if they qualified, they had an 88.1 percent probability of advancing to the semifinals; if they did, they had an 82.0 percent probability of progressing to the final; if they did, they had a 74.0 percent probability of winning the whole competition. It is clear that the lower probability of winning the playoffs is not caused by Sparta 
Praha not being a clear favorite in each round, but rather by even small probabilities of elimination accumulating over multiple rounds.

There are two obvious ways of increasing the probability of the best team winning the playoffs; first, raise the number of matches in each round (this would increase the probability of the better team advancing to the next round); second, lower the number of rounds. Since both spectators and competition organizers seem to prefer higher seasonal uncertainty, there is probably no demand for such changes, but they would be either impractical or have only a moderate impact anyway. For example, the quarterfinals, semifinals, and final would have to use best-of-fifteen instead of best-ofseven system (i.e. eight instead of four wins to eliminate the other team) to approximately neutralize the impact of the playoffs on seasonal uncertainty. This would make the playoff stage much longer, decrease the importance of a single match, and likely lower the interest of spectators. Similarly, if only top four teams qualified for the playoffs, the regular season finish would be less interesting and the best team would still have a lower probability of winning the playoffs than winning the regular season. ${ }^{9}$

${ }^{9}$ These results are based on additional simulation sets using the same team strengths and seasons, but different tournament designs. 


\section{CONCLUSION}

As shown in the previous section, the additional playoff stage lowers the probability that the best team becomes a champion, raises this probability for the other teams, and thus increases seasonal uncertainty. This makes the Extraliga competition more attractive - the supporters of the best team cannot be so sure about the final outcome and the fans of weaker teams have a stronger hope of celebrating the championship title. The fact that securing a higher seed significantly increases championship chances makes the regular season finish interesting for fans of almost all teams. The higher seasonal uncertainty is also likely to translate into a more even distribution of all types of revenues and thus a higher competitive balance. In a positive feedback loop, this further increases seasonal uncertainty. Therefore, the Extraliga tournament design - at least in terms of promoting uncertainty of outcome - seems to be close to ideal. A similar conclusion is also likely to hold for other similarly organized competitions, such as other top European ice hockey competitions, the NHL and the KHL. On the other hand, competitions such as top European soccer leagues could profit from incorporating a properly planned playoff stage into their tournament design. 


\section{REFERENCES}

Buraimo, Babatunde, and Rob Simmons. "Do Sports Fans Really Value Uncertainty of Outcome? Evidence from the English Premier League." International Journal of Sport Finance, no. 3 (2008): 146-155.

Coates, Dennis, and Brad R. Humphreys. "Game Attendance and Competitive Balance in the National Hockey League." University of Alberta Department of Economics Working Paper 2011-08, June 2011.

Dixon, Mark J., and Stuart G. Coles. "Modelling Association Football Scores and Inefficiencies in the Football Betting Market." Journal of the Royal Statistical Society. Series C (Applied Statistics) 46, no. 2 (1997): 265-280.

García, Jaume, and Plácido Rodríguez. "Sports Attendance: A Survey of the Literature 1973-2007." Rivista di Diritto ed Economia dello Sport, no. 2 (September 2009): 111-151.

Goddard, John. "Regression Models for Forecasting Goals and Match Results in Association Football." International Journal of Forecasting, no. 21 (2005): 331-340.

Humphreys, Brad R. "Alternative Measures of Competitive Balance in Sports Leagues." Journal of Sports Economics 3, no. 2 (May 2002): 133-148.

Maher, M J. "Modelling association football scores." Statistica Neerlandica 36, no. 3 (1982): 109-118.

Neale, Walter C. "The Peculiar Economics of Professional Sports: A Contribution to the Theory of the Firm in Sporting Competition and in Market Competition." The Quarterly Journal of Economics 78, no. 1 (February 1964): 1-14.

Pawlowski, Tim, and Oliver Budzinski. "The (monetary) value of competitive balance for sport consumers: A stated preferences approach to European professional football." Ilmenau Economics Discussion Paper, Ilmenau University of Technology, Institute of Economics, No. 77, 2012.

Rottenberg, Simon. "The Baseball Players' Labor Market." Journal of Political Economy 64, no. 3 (June 1956): 242-258.

Rue, Håvard, and Øyvind Salvesen. "Prediction and Retrospective Analysis of Soccer Matches in a League." Journal of the Royal Statistical Society. Series D (The Statistician) 49, no. 3 (2000): 399-418.

Scarf, Philip A, and Muhammad Mat Yusof. "A numerical study of tournament structure and seeding policy for the soccer World Cup Finals." Statistica Neerlandica 65, no. 1 (2011): 43-57. 
Scarf, Philip A, Muhammad Mat Yusof, and Mark Bilbao. "A numerical study of designs for sporting contests." European Journal of Operational Research, no. 198 (2009): 190-198.

Szemberg, Szymon, Martin Merk, and Adam Steiss. "What's the value of being 1st?" International Ice Hockey Federation. March 2, 2012. http://www.iihf.com/nc/home-of-hockey/news/newssingleview/recap/6495.html (accessed February 15, 2013).

Szymanski, Stefan. "Income Inequality, Competitive Balance and the Attractiveness of Team Sports: Some Evidence and a Natural Experiment from English Soccer." The Economic Journal, no. 111 (February 2001): F69-F84.

Szymanski, Stefan. "The Economic Design of Sporting Contests." Journal of Economic Literature 41, no. 4 (December 2003): 1137-1187.

Szymanski, Stefan, and Stefan Késenne. "Competitive Balance and Gate Revenue Sharing in Team Sports." The Journal of Industrial Economics 52, no. 1 (March 2004): 165-177.

Thomas, Andrew C. "Inter-arrival Times of Goals in Ice Hockey." Journal of Quantitative Analysis in Sports 3, no. 3 (2007).

Vrooman, John. "A General Theory of Professional Sports Leagues." Southern Economic Journal 61, no. 4 (April 1995): 971-990. 


\section{APPENDiX A: EsTimATING TEAM STRENGTHS}

This section describes how team strengths are estimated from the actual regular season results. This is done by setting the total expected numbers of regulation-time goals scored and conceded by each team in its home and away matches equal to the corresponding actual values in a given season. For example, team 1 is expected to score $2{ }^{*}$ HomeAttack $_{1}{ }^{*}$ AwayDefense $_{2}$ goals in its two home matches against team 2, $2 *$ HomeAttack $_{1} *$ AwayDefense $_{3}$ goals in its two home matches against team $3 \ldots 2 *$ HomeAttack $_{1} *$ AwayDefense $_{14}$ goals in its two home matches against team 14 . The sum of these expressions is set equal to the total number of goals that team 1 actually scored in all its home matches. Eventually, this leads to the following set of 14 equations (one equation for each team $i$, where $i=1 \ldots 14$ ):

$$
2 * \text { HomeAttack }_{i} * \sum_{j=1 \ldots 14, j \neq i} \text { AwayDefense }_{j}=\text { Total home goals scored by team } i
$$

Similar sets of equations are also put together for goals conceded in home matches and goals scored and conceded in away matches:

$$
\begin{gathered}
2 * \text { HomeDefense }_{i} * \sum_{j=1 \ldots 14, j \neq i} \text { AwayAttack }_{j}=\text { Total home goals conceded by team } i \\
2 * \text { AwayAttack }_{i} * \sum_{j=1 \ldots 14, j \neq i} \text { HomeDefense }_{j}=\text { Total away goals scored by team } i \\
2 * \text { AwayDefense }_{i} * \sum_{j=1 \ldots 14, j \neq i} \text { HomeAttack }_{j}=\text { Total away goals conceded by team } i
\end{gathered}
$$

In the resulting system, there are 56 equations and 56 variables; however, the equations are not independent, since the total number of home goals scored by all teams equals the total number of away goals conceded by all teams and the total number of home goals conceded by all teams equals the total number of away goals scored by all teams. Therefore, there are infinitely many solutions; these can be obtained from each other by multiplying all attack parameters by a positive number and dividing all defense parameters by the same number. Because all solutions provide exactly the same match predictions, this is not a problem and any solution will do. Another option, used in Maher (1982), would be to impose additional constraints on parameter values. 


\section{APPENDIX B: MODEL VERIFICATION}

This section shows that the model produces realistic results by comparing the aggregate simulation statistics against the corresponding actual results for each season or all seasons together. First, the total number of regular season goals scored in each season (including extra time) is compared against the total number of goals in the corresponding set of 1,000,000 simulations based on that season. Since team strengths are actually estimated from total goals, the median ( $50^{\text {th }}$ percentile) number of goals in a simulated season should be very close to the actual total number of goals (the only reason for these two numbers being different is a random number of matches going into extra time in the actual season). This comparison is presented in Table 1.

\begin{tabular}{|c|c|c|c|c|}
\hline \multirow{2}{*}{ Season } & \multicolumn{4}{|c|}{ Total goals (including extra time) } \\
\cline { 2 - 4 } & \multicolumn{2}{|c|}{ Simulation percentiles } & Actual \\
& $5^{\text {th }}$ & $50^{\text {th }}$ & $9^{\text {th }}$ & value \\
\hline $2006 / 07$ & 2051 & 2126 & 2202 & 2123 \\
\hline $2007 / 08$ & 1966 & 2039 & 2113 & 2057 \\
\hline $2008 / 09$ & 2024 & 2099 & 2175 & 2096 \\
\hline $2009 / 10$ & 1986 & 2060 & 2135 & 2057 \\
\hline $2010 / 11$ & 1950 & 2023 & 2096 & 2012 \\
\hline $2011 / 12$ & 1924 & 1996 & 2070 & 1995 \\
\hline
\end{tabular}

TABle 1: SimUlated VS. ACTUAL TOTAL NUMBER OF GOALS (INCLUDING EXTRA TIME)

In each season, the difference between the simulated median and the actual number of total goals is less than 1 percent; the average difference across all seasons is 0.02 percent. Therefore, the individual match model does not seem to be biased in terms of the total number of goals (including extra time).

In the second test, the simulated relative frequency of each type of match result (home team regulation/extra time win/loss) in a regular season is compared against the actual relative frequency. Because there are only 364 matches in one season, all seasons are pooled together for the total number of 2,184 matches to increase the test power. The relative frequencies across all seasons are shown in Table 2.

\begin{tabular}{|c|c|c|c|c|}
\cline { 2 - 5 } \multicolumn{1}{c|}{} & \multicolumn{2}{c|}{ Home team win } & \multicolumn{2}{c|}{ Home team loss } \\
\cline { 2 - 5 } \multicolumn{1}{c|}{} & Regulation & Extra time & Extra time & Regulation \\
\hline Simulated relative frequency & 0.4915 & 0.1231 & 0.0988 & 0.2866 \\
\hline Actual relative frequency & 0.5023 & 0.1200 & 0.1058 & 0.2720 \\
\hline
\end{tabular}

TABle 2: Simulated VS. ACTUAL RElative FREQUEnCiES OF MATCH RESUlt TyPES, N = 2,184 
For each type of result, the difference between the simulated and the actual relative frequency is within 2 percentage points and the distribution of actual result types is not statistically significantly different from the simulated distribution at $\alpha=0.05$ (chi-square goodness-of-fit test, $\mathrm{p}$-value $=$ 0.334). Consequently, the model does not seem to be biased in terms of the result type.

Third, the total minimum and maximum numbers of points in each regular season (i.e. the points obtained by the team that finished last and the winner) ${ }^{10}$ are compared against the total maximum and minimum numbers of points in the corresponding set of $1,000,000$ simulations. Table 3 presents simulated point percentiles and the corresponding actual values.

\begin{tabular}{|c|c|c|c|c|c|c|c|c|}
\hline \multirow{3}{*}{ Season } & \multicolumn{4}{|c|}{ Minimum points } & \multicolumn{4}{|c|}{ Maximum points } \\
\hline & \multicolumn{3}{|c|}{ Simulation percentiles } & \multirow{2}{*}{$\begin{array}{c}\text { Actual } \\
\text { value }\end{array}$} & \multicolumn{3}{|c|}{ Simulation percentiles } & \multirow{2}{*}{$\begin{array}{l}\text { Actual } \\
\text { value }\end{array}$} \\
\hline & $5^{\text {th }}$ & $50^{\text {th }}$ & $95^{\text {th }}$ & & $5^{\text {th }}$ & $50^{\text {th }}$ & $95^{\text {th }}$ & \\
\hline $2006 / 07$ & 20 & 31 & 44 & 34 & 97 & 105 & 116 & 100 \\
\hline $2007 / 08$ & 25 & 38 & 51 & 40 & 99 & 109 & 120 & 106 \\
\hline $2008 / 09$ & 48 & 58 & 66 & 59 & 90 & 97 & 107 & 93 \\
\hline $2009 / 10$ & 44 & 54 & 62 & 58 & 96 & 105 & 116 & 106 \\
\hline $2010 / 11$ & 33 & 46 & 56 & 41 & 96 & 103 & 114 & 96 \\
\hline $2011 / 12$ & 43 & 53 & 61 & 61 & 99 & 109 & 121 & 107 \\
\hline
\end{tabular}

TABle 3: Simulated vs. ACTUAL Minimum/maximum POINTS

The average difference between the simulated median number of points and the actual number is 3.75 points (a little more than the difference between winning and losing a single match). Each interval between the $5^{\text {th }}$ and $95^{\text {th }}$ point percentiles can be thought of as a 90 -percent confidence interval on the prediction of the actual number of points; there are 12 such intervals and the actual value lies on the interval boundary in two cases and never outside. Therefore, the simulation also seems to produce realistic regular season point distributions.

The last test compares the simulated relative frequencies of best-of-seven playoff series results (i.e. the quarterfinals + the semifinals + the finals) from the point of view of the higher-seeded team against the actual relative frequencies. Because there are only seven such results per season, all seasons are again pooled together for the total number of 42 series results. The simulated and actual relative frequencies across all seasons are shown in Table 4 (the first number in the result represents the number of matches won by the higher-seeded team and the second number represents the number of matches won by the lower-seeded team).

\footnotetext{
${ }^{10}$ In the 2010/11 season, three teams were deducted points due to invalid player registration forms. These
} deductions are not taken into account in this test. 


\begin{tabular}{|c|c|c|c|c|c|c|c|c|}
\cline { 2 - 8 } \multicolumn{1}{c|}{} & \multicolumn{8}{c|}{ Quarter/semi/final playoff series result } \\
\cline { 2 - 9 } & $4-0$ & $4-1$ & $4-2$ & $4-3$ & $3-4$ & $2-4$ & $1-4$ & $0-4$ \\
\hline $\begin{array}{c}\text { Simulated relative } \\
\text { frequency }\end{array}$ & 0.1004 & 0.2116 & 0.1512 & 0.1950 & 0.0963 & 0.1393 & 0.0679 & 0.0383 \\
\hline $\begin{array}{c}\text { Actual relative } \\
\text { frequency }\end{array}$ & 0.0714 & 0.2857 & 0.1190 & 0.2143 & 0.0714 & 0.1429 & 0.0476 & 0.0476 \\
\hline
\end{tabular}

Table 4: Simulated VS. ACtUAl Relative FREQUenCies of Playoff SERIES RESUlts, $\mathrm{N}=42$

The highest difference between the simulated and actual relative frequency is 7 percentage points (4-1 result); this result was predicted to happen about 9 times, but it actually happened 12 times. However, this is completely natural given the small sample size; the actual result type distribution is not statistically significantly different from the simulated distribution at $\alpha=0.05$ (chi-square goodness-of-fit exact test, $\mathrm{p}$-value $=0.937$ ). It is also possible to look at the simulated versus the actual relative frequency of the higher-seeded team eliminating the lower-seeded team (4-0 + 4-1 + 4-2 + 4-3 results); again, the actual relative frequency of 0.6905 is not statistically significantly different from the simulated relative frequency of 0.6582 at $\alpha=0.05$ (two-tailed t-test, p-value $=$ 0.661). 